\title{
Permanent Nonselective His Bundle Pacing in an Adult with L- Transposition of the Great Arteries and Complete AV Block
}

Nonselective His Bundle Pacing LTGA

Adam C. Kean, MD, MPH - Corresponding Author

Assistant Professor of Clinical Pediatrics

Department of Pediatrics, Division of Pediatric Cardiology

Pediatric Electrophysiology

Indiana University School of Medicine

705 Riley Hospital Drive

Riley Research Room 127

Indianapolis, IN 46202-5225

317-274-8906 (T); 317-274-4022 (F)

akean@iu.edu

No relevant conflicts of interest

W. Aaron Kay, MD

Assistant Professor of Medicine, Indiana University

Director, Adult Congenital Heart Disease Program

Krannert Institute of Cardiology

Indiana University Health Physicians

No relevant conflicts of interest

Jyoti K. Patel, MD

Assistant Professor of Clinical Pediatrics

Indiana University School of Medicine

Section of Pediatric Cardiology

No relevant conflicts of interest

John M. Miller, MD

Professor of Medicine, Indiana University School of Medicine

Director, Clinical Cardiac Electrophysiology, Indiana University Health

No relevant conflicts of interest

Gopi Dandamudi, MD

Assistant Professor of Medicine, Indiana University

Director, Atrial Fibrillation Program

Krannert Institute of Cardiology

This is the author's manuscript of the article published in final edited form as:

Kean, A. C., Kay, W. A., Patel, J. K., Miller, J. M. and Dandamudi, G. (2017), Permanent nonselective His bundle pacing in an adult with L-transposition of the great arteries and complete AV block. Pacing Clin Electrophysiol. Accepted Author Manuscript. http://dx.doi.org/10.1111/pace.13107 
Indiana University Health Physicians

Medtronic-Speaker, Bohringer-Ingelheim-Speaker

Abstract:

We report the placement of a permanent transvenous nonselective His bundle pacing lead in conjunction with a transvenous pacemaker/ICD in an adult with Levo-Transposition of the Great Arteries (L-TGA) and a stenotic coronary sinus (CS) ostium (os), which would not accommodate a transvenous LV pacing lead. Nonselective His bundle pacing provided a nearly identical ventricular activation pattern in this previously unpaced patient. Many L-TGA patients will have an eventual need for permanent pacing and, given the challenges of CS cannulation, His bundle pacing may represent a preferred modality rather than pure morphologic LV pacing or surgical systemic ventricular lead placement to achieve optimal electrical synchrony.

Key Words: L-TGA, Congenitally corrected TGA, Complete heart block, Non-selective His bundle pacing, Adult congenital heart disease.

Introduction:

L-TGA, sometimes referred to as "congenitally corrected transposition of the great arteries," (due to the restoration of pulmonary and systemic circulations in series rather than in parallel) is a congenital cardiac anomaly in which the embryological looping of the ventricles is reversed such that the morphological LV (identified by its paucity of trabeculae, less apically positioned atrioventricular valve, lack of connection from the atrioventricular valve to the septal surface, and bullet shape) sits anteriorly and to the right beneath the right atrium with outflow to the pulmonary valve and the morphological RV (identified by significant coarse trabeculations, a moderator band, a more apically positioned atrioventricular valve, the presence of atrioventricular valve connections to the septum, and triangle shape) sits posteriorly and to the left beneath the left atrium with outflow to the aortic

This article is protected by copyright. All rights reserved. 
valve [1]. In the setting of this reversed looping, the cardiac conduction system often suffers with congenital or progressive atrioventricular (AV) dissociation distal to the AV node.

The incidence of this malformation is unclear due to its varied natural history [2] ranging from a complete lack of symptoms with normal life expectancy to neonatal congestive heart failure in the setting of complete AV block and a dilated systemic right ventricle leading to severe systemic tricuspid valve regurgitation.

We present a patient with progressive right ventricular dilation, subsequent tricuspid valve replacement secondary to severe regurgitation with a dilated left atrium, longstanding persistent atrial fibrillation (AF) and progressive AV block who described increased symptoms of fatigue with moderate activity. The CS os appeared stenotic on magnetic resonance imaging (MRI), and could not be cannulated from the inferior (IVC) or superior vena cava (SVC). With the identification of the His bundle electrogram, the option of permanent His bundle pacing with morphologic LV pacing back-up was available. This strategy was previously described in a pediatric patient [3].

We report the successful placement of permanent nonselective His bundle pacing system in an adult patient with L-TGA.

\section{Case:}

Our patient is a 38-year-old male undergoing routine follow-up evaluation for his underlying L-TGA and mechanical systemic (tricuspid) valve that was replaced 10 years prior for severe regurgitation [Figure 1]. There was no evidence of surgical AV block following the mechanical valve placement though epicardial pacing leads were placed and tunneled to the abdomen at the time of that operation given the high incidence of post-operative heart block in patients with L-TGA. The patient describes minimal symptoms with shortness of breath during moderate activity that bothers his wife more than it does him. He works full time in education that requires significant activity, working on 
his feet throughout the day. He is maintained on warfarin for INR goal 3.0, atorvastatin 40mg PO daily, and Lisinopril $5 \mathrm{mg}$ PO daily. His exam is notable for being mildly over-weight at $180 \mathrm{~cm}, 85 \mathrm{~kg}$, BMI 26.2, normotensive at 133/78, resting bradycardia at $49 \mathrm{bpm}$, and $100 \%$ saturated on room air. His lung exam is unremarkable. Cardiac exam reveals a normal apical impulse, no lifts or heaves, a regular rhythm, mechanical first heart sound, and a fixed split second heart sound with no murmur. Neither jugular venous nor abdominal distension were present.

A 12 lead ECG six months' prior, showed atrial fibrillation (AF) with a regular ventricular escape at 50 bpm, right axis deviation, and deep Q waves in V1 and V2 consistent with old anteroseptal infarct [Figure 2A]. A Holter monitor completed just prior to the evaluation showed AF with variable AV conduction, intraventricular conduction delay (IVCD) and periodic complete AV block with IVCD escape. Heart rate ranged from $33-74 \mathrm{bpm}$ with a mean of $44 \mathrm{bpm}$. A cardiac MRI showed moderate systemic right ventricular dysfunction with an ejection fraction of $35 \%$ and moderate dilation of the right ventricle with an end diastolic volume of $352 \mathrm{ml}$. [Figure 3] There was normal left ventricle systolic function with an ejection fraction of $67 \%$. The left atrium was moderately dilated. CS anatomy appeared normal though the os was difficult to visualize and likely significantly stenotic and there was significant dilation of the coronary veins [Figure 3]. After review of these findings and discussion, he was referred first for AF ablation followed by planned transvenous biventricular pacemaker/implantable cardioverter-defibrillator (ICD) placement. The indication for ICD placement was for primary prevention against sudden cardiac death in the setting of low systemic ventricular ejection fraction.

Seven weeks after initial presentation, our patient presented for EP study and ablation of persistent $A F$, atrial cycle length of $<100$ msec with junctional escape of $1200 \mathrm{msec}$. QRS duration was 120 msec with a left bundle brand block (LBBB) morphology and axis of 30 degrees. Access to the left 
atrium was obtained and mapping of the pulmonary veins demonstrated no particular pulmonary vein as a likely AF source. Aided by electroanatomic mapping, wide area circumferential ablation was completed to isolate the left and right pulmonary veins. Since AF persisted, focal impulse and rotor modulation (FIRM) ablation was then completed with right and left atrial sources eliminated. Cardioversion then restored sinus rhythm with complete AV block [Figure 2B]. A His bundle recording could not be reliably obtained. A proximal right-sided left bundle potential to QRS interval was $33 \mathrm{msec}$. Of note, the CS could not be cannulated beyond the os due to possible stenosis of the os acquired during valve replacement, Thebesian valve protection, or difficult anatomical angles from the IVC. He recovered from the procedure without incident though described increased fatigue in the setting of sinus rhythm and complete AV block.

Seven weeks following ablation, our patient presented for placement of a biventricular transvenous pacemaker/ICD due to persistent complete AV block and a systemic ventricular ejection fraction of $<35 \%$. 12-lead ECG showed sinus rhythm with completed AV dissociation and junctional escape with LBBB morphology. There was left atrial hypertrophy with an atrial rate $71 \mathrm{bpm}$, ventricular rate 48 bpm, and QRS duration 120 msec.

Procedural sedation was initiated and a left infraclavicular incision was made followed by left axillary venous access. A single coil ICD lead was placed at the septal apex of the right-sided morphological left ventricle and secured into place with adequate sensing and pacing parameters. A second lead was placed in the anterior right atrium and secured into place with adequate sensing and pacing parameters. In turning our attention to systemic ventricular/CS lead placement, review of the patient's MRI demonstrated presence of the CS although the os appeared stenotic. Advancement of an introducer wire was not successful. This was followed by efforts with a $6 \mathrm{~F}$ quadripolar electrode 
catheter adding further insight into CS os vs tricuspid annulus location. The os was entered although the catheter could not be advanced into the main body of the CS.

After considerable effort at CS cannulation, it was decided to attempt a His bundle recording with the catheter. Though characteristic His bundle electrogram was not identified, a proximal bundle brand potential (closer to the ventricular electrogram and with a diminutive atrial electrogram preceding it) in the characteristic anterior position consistent with the description by Anderson et al [3]. Pacing at that location captured the ventricle with a QRS complex identical to the junctional escape complex with minimal local myocardial capture and fusion (non-selective His bundle pacing [Figure 2C]). Given this finding and a baseline QRS duration more favorable than with RV pacing (>> $160 \mathrm{msec}$ ), it was decided to place a permanent lead at this location in addition to the alreadyplaced ICD lead. A Medtronic bipolar model 3830 lead was positioned at the location of the recorded potential. Securing the lead along the anterior septum adjacent to the mitral valve annulus required multiple attempts though was eventually successful with adequate sensing and pacing parameters [Figures 1 and 4]. The proximal lead was secured in the pocket and all leads connected to the generator. The pocket was irrigated and closed per standard protocol. He was programmed DDD 50 - 150/min with the "right ventricle" (morphological left ventricle) behind the "left ventricle" (His) at $80 \mathrm{msec}$ in the event of nonselective His bundle lead capture failure.

The following morning, the patient was recovering well and his rhythm showed A-sense, V-pace with near baseline QRS complex indicative of nonselective His bundle pacing. The QRS complex showed subtle changes in the precordial and lateral limb leads. These changes are likely due to fused activation of the His bundle, physiological left bundle, and ventricular septal myocardium. This accounts for the near immediate QRS following pacing spike. Additionally the paced QRS complex is 
a small amount wider than the baseline, close to $160 \mathrm{msec}$. Lead capture threshold was $1.25 \mathrm{~V}$ at $1.0 \mathrm{msec}$, with a pacing impedance of 342 ohms. Two weeks later, he presented to follow-up his AF ablation and was noted to be feeling well and was in sinus rhythm with consistent atrial tracking and nonselective His bundle capture at $1.25 \mathrm{~V}$ at $0.4 \mathrm{msec}$.

\section{Discussion:}

This case represents the first description of distal His bundle pacing in a patient with L-TGA. The natural history of patients with uncomplicated L-TGA is progressive AV conduction loss in a greater frequency than the normal population [2]. Anatomic descriptions from pathological specimens describe an anteriorly displaced $A V$ node adjacent to the pulmonary valve annulus with an extended length of His bundle that is not easily seen connecting to the proximal bundle branches; this seems prone to conduction loss [4]. The sub-pulmonary and rightward morphologic LV retains its ribbonlike left bundle while the systemic and leftward, morphologic RV retains its discrete right bundle coursing along the moderator band. We were able to record a distal His bundle potential prior to bifurcation as pacing from this site led to only minimal QRS morphology changes. It remains unclear as to whether lateral right ventricular free wall pacing via the CS would have produced QRS narrowing. The challenges of placing such a lead cannot be understated, eliminating this option. When pacing from the morphological left ventricular apex, however, there was significant QRS widening as compared with the junctional escape. It was therefore preferable to pace with a near native QRS complex, similar to that found in His bundle pacing in patients with normal cardiac anatomy [5]. At present, follow-up functional studies are pending. Given the underlying mild intraventricular conduction delay it may be reasonable to hypothesize that nonselective His bundle pacing with a near-native QRS would be no worse than BiV pacing. Chronic morphologic LV pacing, however, would likely be much worse.

This article is protected by copyright. All rights reserved. 
We were able to demonstrate a reasonable acute lead capture threshold comparable to His bundle pacing in the anatomically normal heart while still providing appropriate ventricular pacing backup.

While chronic threshold data remains to be determined, the procedural feasibility is now demonstrated in this population. Many L-TGA patients will have an eventual need for permanent pacing and, given the challenges of CS cannulation, His bundle pacing may represent a preferred modality rather than pure morphologic LV pacing or surgical systemic ventricular lead placement to achieve optimal electrical synchrony. Further study beginning with a case series and eventual comparison trial may be warranted.

Author contributions:

Adam C. Kean, MD, MPH - Concept, data interpretation, drafting article, critical revision, and approval.

W. Aaron Kay, MD - Data interpretation, literature review, critical revision, and approval.

Jyoti K. Patel, MD - Data interpretation, critical revision, and approval.

John M. Miller, MD - Data interpretation, critical revision, and approval.

Gopi Dandamudi, MD - Concept, data interpretation, drafting article, critical revision, and approval.

\section{References:}

1. Grant R. "The Morphogenesis of Correct Transposition and Other Anomalies of Cardiac Polarity."

Circulation. 1964; 29:71-83.

2. Graham TP, Bernard YD, Mellen BG, Celermajr D, Baumgartner H, Cetta F, Conolly HM, et al. "Long-term outcome in congenitally corrected transpostion of the great arteries: a multiinstitutional study." J Am Coll Cardiol. 2000. July; 36 (1):255 - 61.

This article is protected by copyright. All rights reserved. 
3. Takemoto M, Nakashima A, Muneuchi J, Yamamura KI, Shiokawa Y, Sunagawa K, and Tominaga R. "Para-Hisian Pacing for a Pediatric Patient with a Congenitally Corrected Transposition of the Great Arteries (SLL)." Pacing and Clinical Electrophysiology. 2009. September; 33: e4-e7.

4. Anderson RH, Becker AE, Arnold R, Wilkinson JL. "The Conducting Tissues in Congenitally Corrected Transposition." Circulation. 1974. November; 50(5):911-23.

5. Sharma PS, Dandamudi G, Naperkoski A, Oren JW, Storm RH, Ellenbogen KA, Vijayaraman P. "Permanent His-bundle Pacing is Feasible, Safe, and Superior to Right Ventricular Pacing in Routine Clinical Practice." Heart Rhythm. 2015. February; 12 (2):305 - 12.

Figure 1. LTGA Patient Heart Diagram

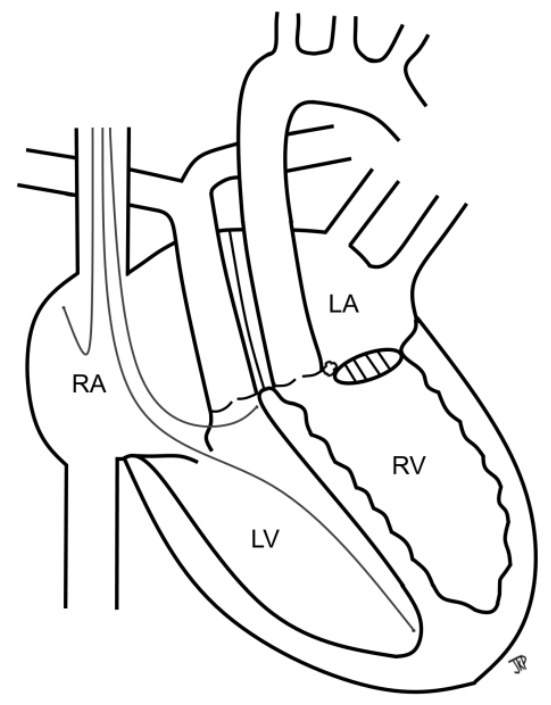

Figure 2. A. Baseline 12 lead ECG.

This article is protected by copyright. All rights reserved. 


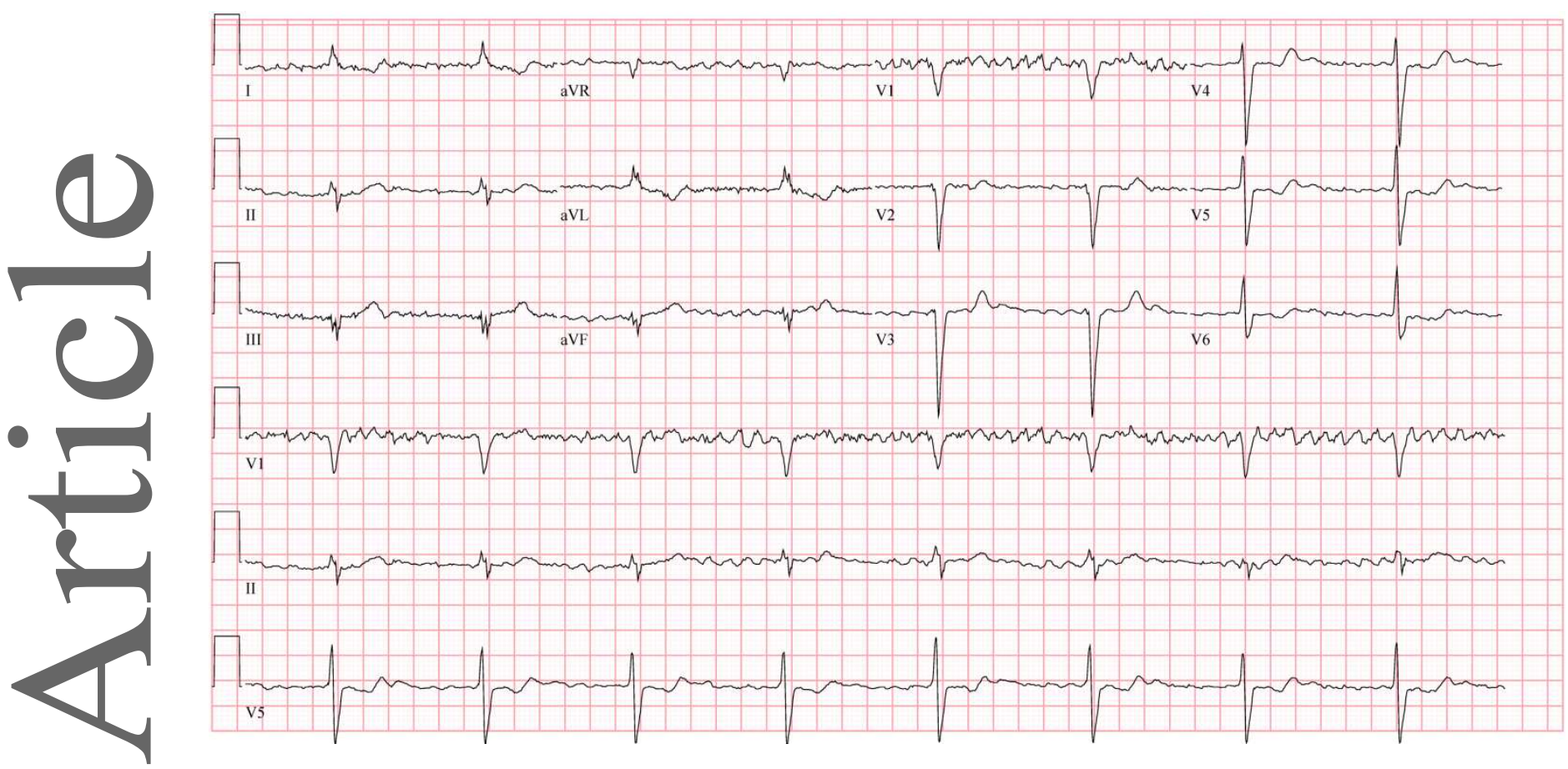

B. ECG following AF ablation.
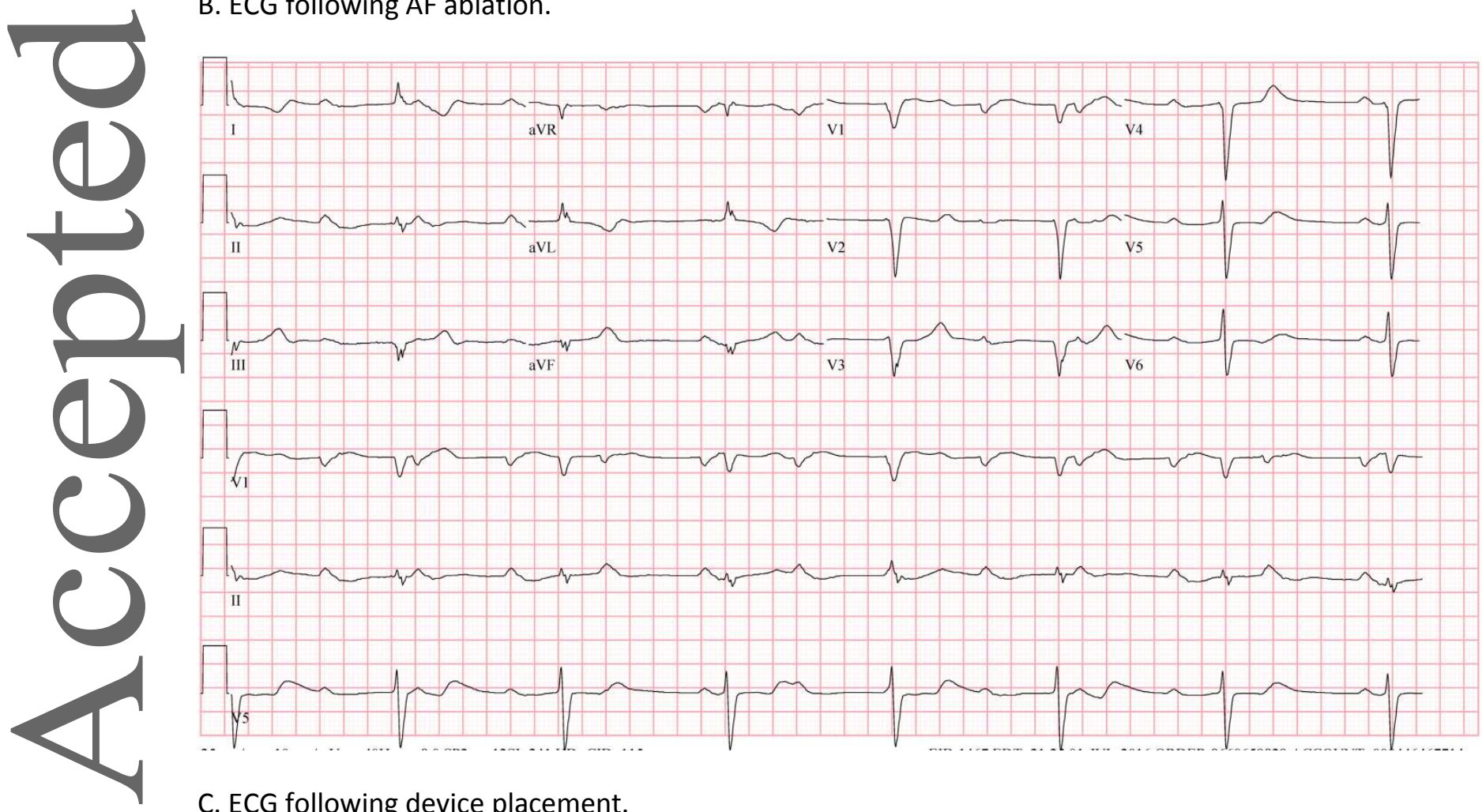

C. ECG following device placement.

This article is protected by copyright. All rights reserved. 


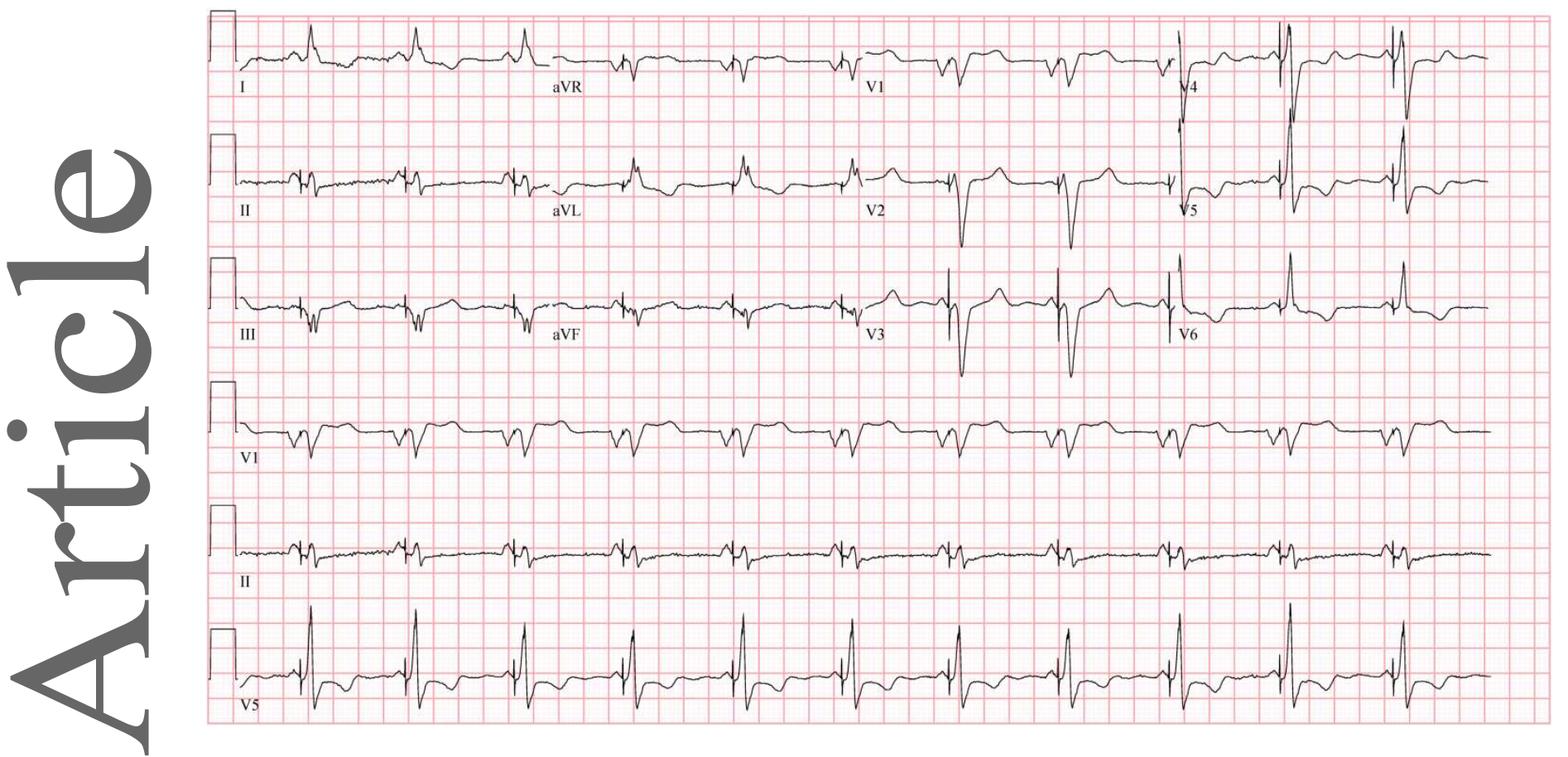

Figure 3. Cardiac MRI showing CS anatomy. Note sizeable sinus body though no clear os.

This article is protected by copyright. All rights reserved. 


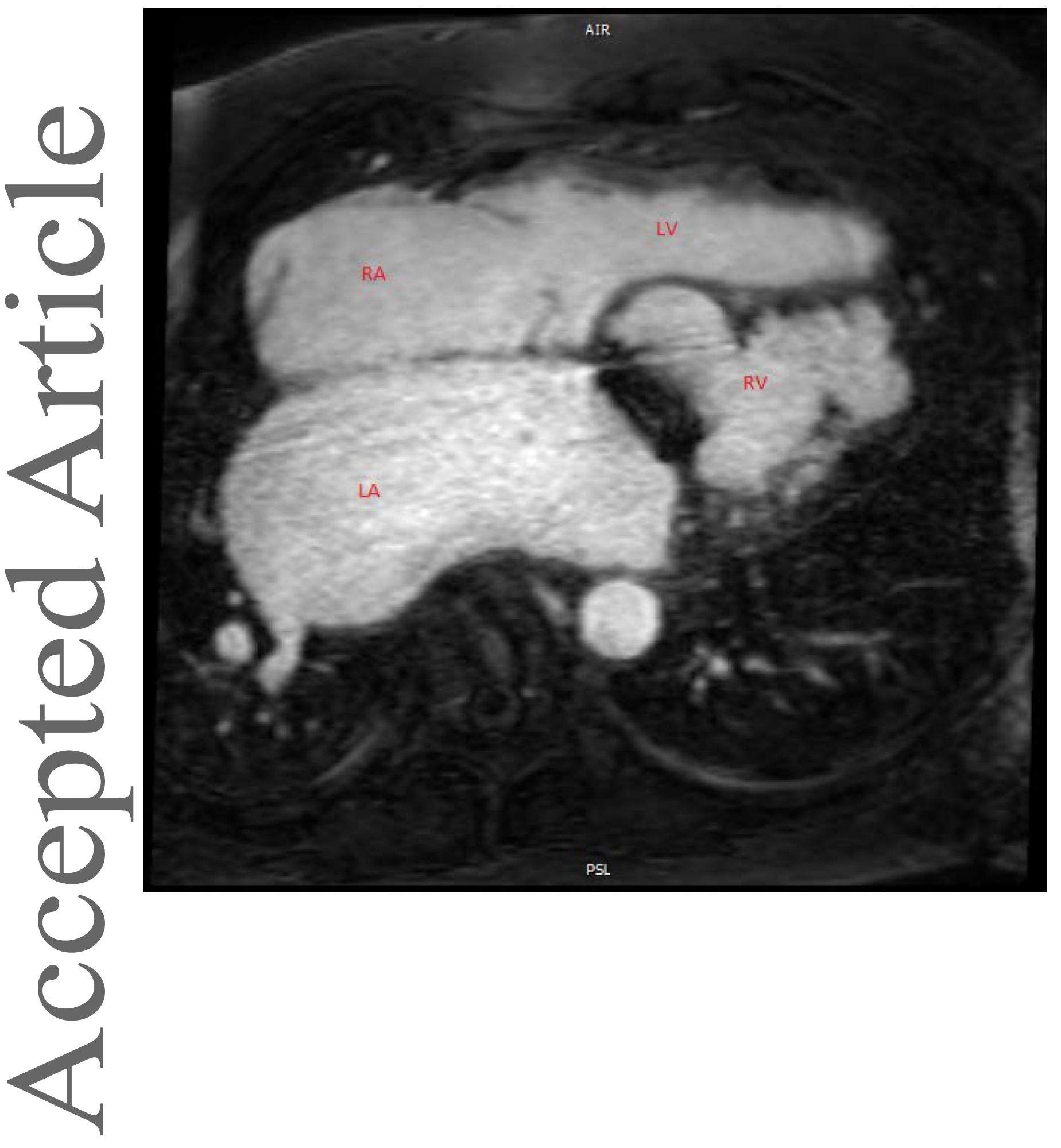

This article is protected by copyright. All rights reserved. 


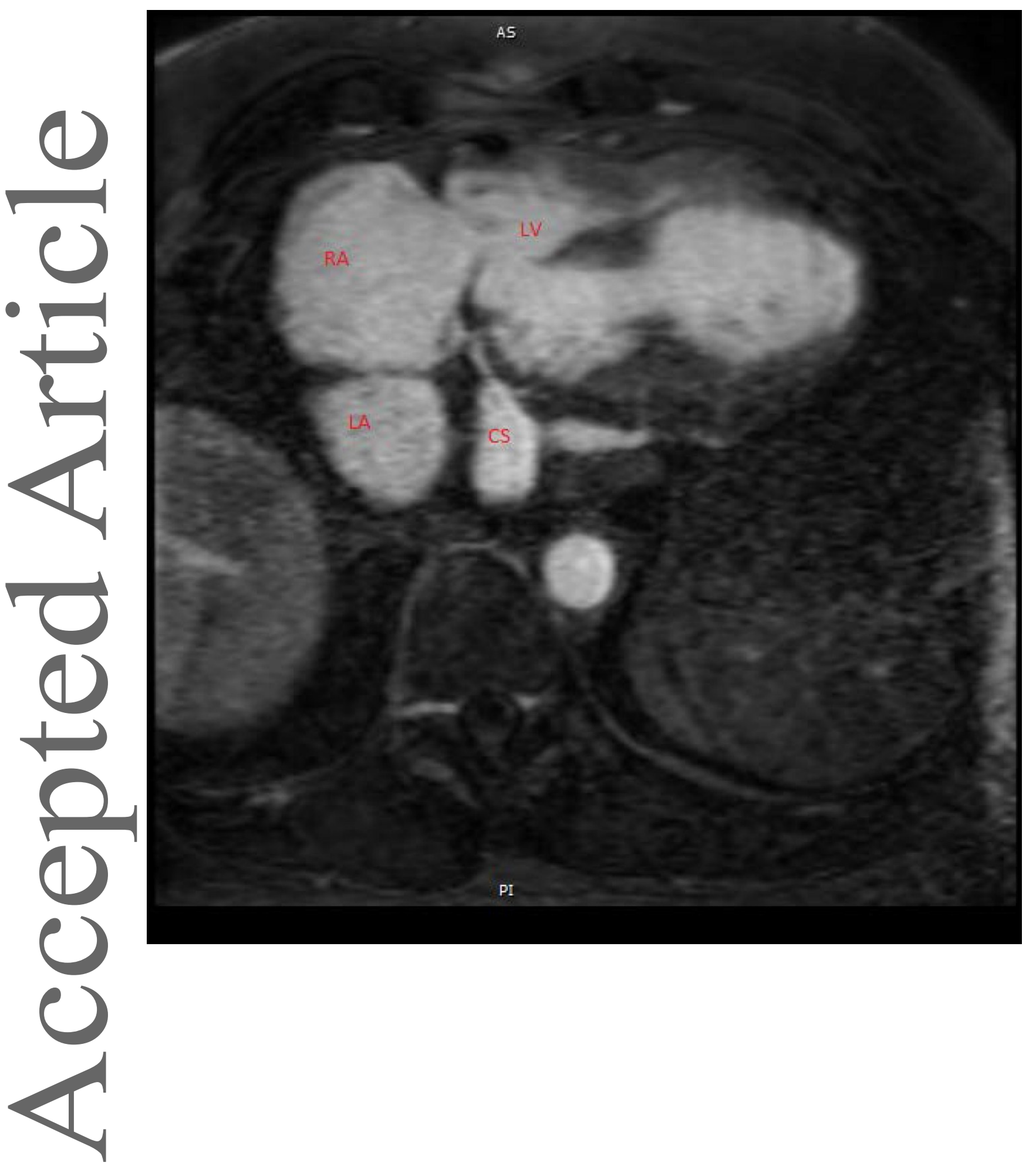

This article is protected by copyright. All rights reserved. 


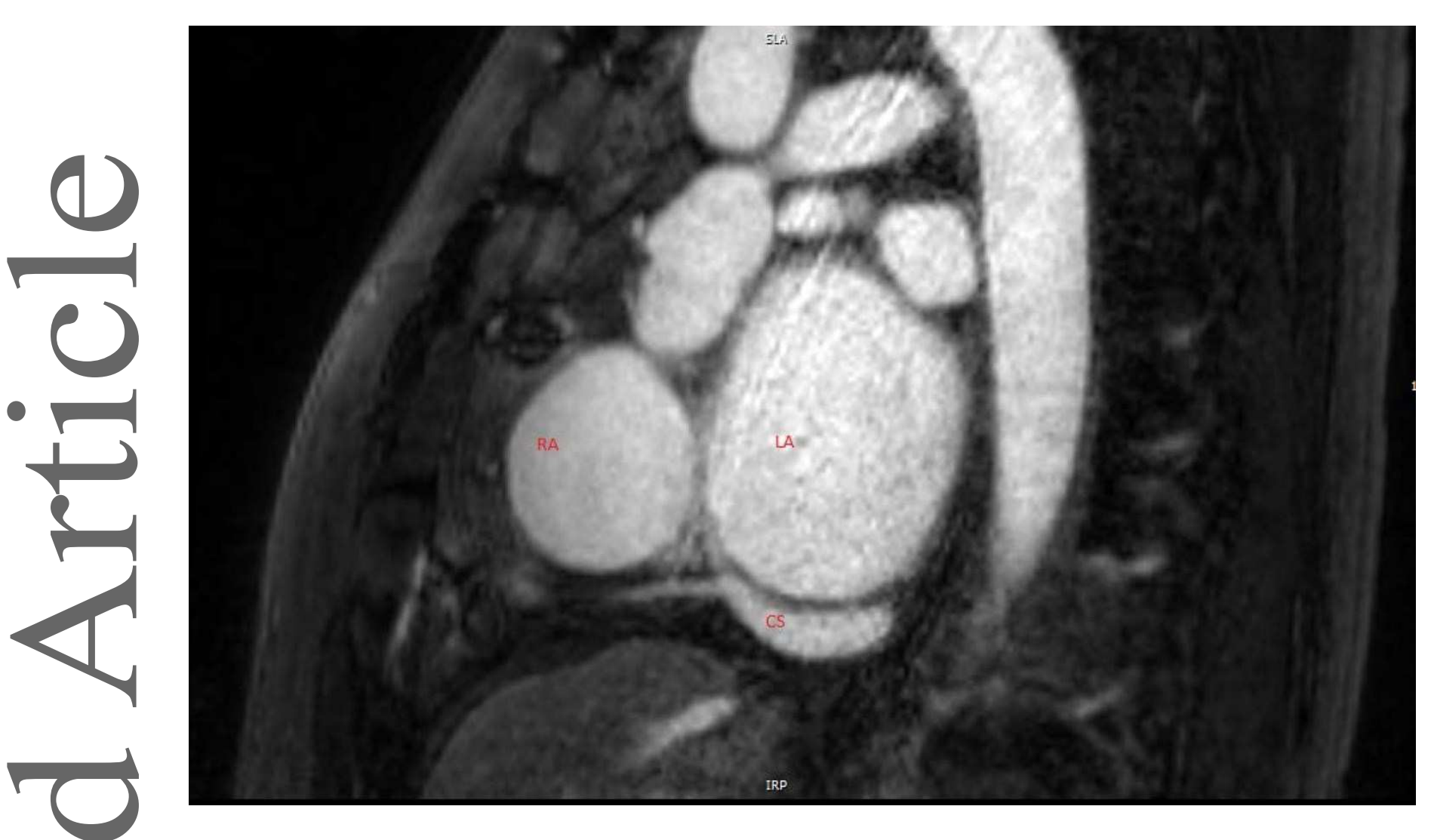

Figure 4. Final chest radiograph showing lead placement.
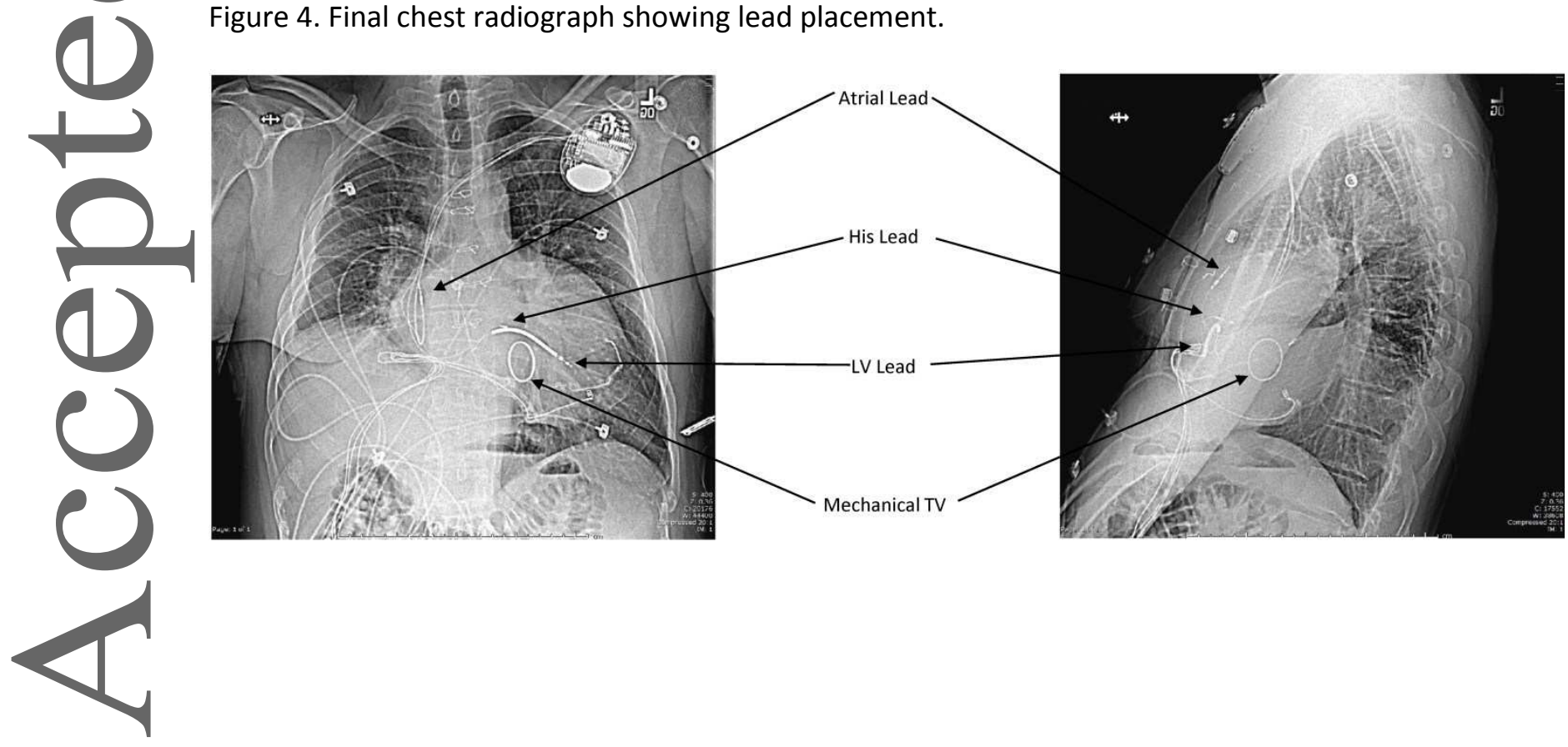

This article is protected by copyright. All rights reserved. 